\title{
Estudos de crustáceos decápodes com isótopos estáveis: Análise bibliométrica entre 2010 e 2020
}

\author{
Studies of decapod crustaceans with stable isotopes: \\ Bibliometric analysis between 2010 and 2020
}

\author{
Keltony de Aquino Ferreira ${ }^{1 *}$, Adriane Araújo Braga ${ }^{2}$, \\ Ana Paula Madeira Di Beneditto ${ }^{1}$
}

\begin{abstract}
RESUMO
Este trabalho tem como objetivo apresentar análise bibliométrica sobre a utilização de isótopos estáveis em estudos com decápodes na última década (2010-2020). O levantamento de informações reuniu 440 artigos publicados em periódicos indexados e revisados por pares subdivididos em 208 publicações diretas, cujo foco eram decápodes, e 232 publicações indiretas, quando esses animais eram apenas parte da amostragem. A quantidade de artigos foi impulsionada por países que possuem maiores investimentos em ciência e tecnologia, tais como Estados Unidos e China. O número de artigos que utilizaram isótopos estáveis como ferramenta de estudo para decápodes progrediu na última década. Entretanto, apenas $2 \%$ do total de espécies foram estudadas $(n=278)$, com ênfase na superfamília Penaeoidea e infraordens Brachyura, Caridea e Anomura. As espécies desses táxons possuem elevada importância comercial, o que justifica o maior interesse em estudá-las. As informações disponíveis nos artigos apontam que a maioria dos estudos aborda principalmente a ecologia trófica, utilizando isótopos estáveis de carbono e nitrogênio $\left(\delta^{13} \mathrm{C}\right.$ e $\left.\delta^{15} \mathrm{~N}\right)$.
\end{abstract}

Palavras-chave: Crustacea; Decapoda; Isótopos estáveis; Ecologia isotópica.

\begin{abstract}
This work aims to present a bibliometric analysis on the use of stable isotopes in studies on decapods in the last decade (2010-2020). The information survey gathered 440 papers published in indexed and peerreviewed journals, subdivided into 208 direct publications, whose focus was decapods, and 232 indirect publications, when these animals were only part of the samplings. The number of papers was boosted by countries that have greater investments in science and technology, such as the United States and China. The number of papers that used stable isotopes as a tool for decapods studies has progressed in the last decade. However, only $2 \%$ of the total species were studied $(n=278)$, with emphasis on the superfamily Penaeoidea and infraorders Brachyura, Caridea and Anomura. The species of these taxa have high commercial importance, which justifies the greater interest in studying them. The information available in the papers indicates that most studies mainly address trophic ecology, using stable isotopes of carbon and nitrogen $\left(\delta^{13} \mathrm{C}\right.$ and $\left.\delta^{15} \mathrm{~N}\right)$.
\end{abstract}

Keywords: Crustacea; Decapoda; Stable isotopes; Isotopic ecology.

\footnotetext{
${ }^{1}$ Laboratório de Ciências Ambientais, Centro de Biociências e Biotecnologia, Universidade Estadual do Norte Fluminense Darcy Ribeiro. *E-mail: keltony.aquino@yahoo.com.br ${ }^{2}$ Departamento de Biologia, Centro de Ciências Exatas, Naturais e da Saúde, Universidade Federal do Espírito Santo.
} 


\section{INTRODUÇÃO}

O subfilo Crustacea (filo Arthropoda) compreende cerca de 70.000 espécies descritas, que estão distribuídas em seis classes: Branchiopoda, Remipedia, Cephalocarida, Maxillopoda, Ostracopoda e Malacostraca. Esses invertebrados ocupam uma variedade de habitats, tais como águas continentais, costeiras e oceânicas profundas, bem como ambiente terrestre, desde os trópicos aos polos. Os crustáceos são dominantes no plâncton e no ambiente bentônico, e desempenham papel importante na estruturação dos ecossistemas aquáticos. A ordem Decapoda constitui um dos táxons mais representativos de Crustacea, com cerca de 15.000 espécies, e inclui os camarões, caranguejos, lagostas, lagostins e siris. Os decápodes são caracterizados por possuírem cinco pares de apêndices locomotores (pereiópodos), três pares de maxilípedes e carapaça desenvolvida que recobre a câmara branquial (BRUSCA e BRUSCA, 2007).

O ciclo de vida dos decápodes inclui metamorfose e troca periódica do exoesqueleto pelo processo de ecdise (ou muda). No ambiente marinho, os estágios larvais planctônicos permanecem em águas superficiais ricas em alimentos. O estágio de eclosão varia muito nos decápodes, com alguns organismos liberando seus ovos diretamente na água, e outros incubando nos pleópodes das fêmeas (RUPPERT et al., 2005). À medida que a fase pós larva se desenvolve, pode ocorrer a migração para regiões mais profundas durante o crescimento, na qual ocorrem processos maturação gonadal e desova (TOM et al., 1988; RUPPERT et al., 2005). O ciclo de vida dos decápodes marinhos pode variar, e há espécies que dependem de estuários (maioria), enquanto outras se mantêm somente no ambiente marinho (DALL et al. 1990). As larvas se diferenciam dos animais adultos quanto aos padrões de locomoção, habitat e forma de alimentação, o que confere vantagens na sobrevivência devido à redução da competição intraespecífica (MARAFON-ALMEIDA et al., 2008).

Os hábitos alimentares dos decápodes são bastante diversificados, e há representantes saprófagos, herbívoros, detritívoros, carnívoros e onívoros. Para explorar todas essas estratégias alimentares, os decápodes normalmente utilizam seus primeiros apêndices locomotores (quelípodos), que geralmente são adaptados para auxiliar na alimentação (RUPPERT et al., 2005; BRUSCA e BRUSCA, 2007). Os decápodes desempenham papel importante nas cadeias e redes alimentares, pois como consumidores secundários eles fornecem energia aos níveis tróficos mais elevados à medida que são 
predados (DALL et al., 1990; BRANCO e VERANI, 1997; OLIVIER et al., 2006; BUI e LEE, 2014).

Nos últimos anos, estudos sobre a biologia e ecologia de crustáceos decápodes progrediram consideravelmente devido a sua importância ecossistêmica. Ferramentas como isótopos estáveis têm obtido sucesso em descrever o espaço ecológico das espécies, além de fornecer informações de nichos tróficos para compreensão da estrutura de redes alimentares (ABRANTES et al., 2014; PHILLIPS et al., 2014).

Os isótopos são variantes do mesmo elemento químico que compartilham o número de prótons e diferem dos demais em relação ao número de nêutrons (MARTINELLI et al., 2009). A análise de isótopos estáveis se baseia na análise da razão isotópica (i.e., razão entre isótopos pesados e leves de um elemento) na amostra em comparação com padrões internacionais de valores conhecidos. O atributo de ser estável se refere ao fato desses isótopos não se deterioram em outros elementos devido à estabilidade na combinação de prótons e nêutrons (FRY, 2008; MARTINELLI et al., 2009).

A distribuição natural dos isótopos estáveis nos organismos reflete os processos físico-químicos e metabólicos de forma integrada e, por isso, eles são reconhecidos como traçadores químicos eficientes. O princípio da composição isotópica dos organismos se baseia nos recursos alimentares consumidos e incorporados aos tecidos, após os processos de digestão e excreção, de modo que a composição isotópica refletirá o habitat do organismo ou seus sítios alimentares FRY, 2008). Os isótopos de carbono (C), nitrogênio $(\mathrm{N})$, oxigênio $(\mathrm{O})$, hidrogênio $(\mathrm{H})$ e enxofre $(\mathrm{S})$ têm elevada abundância natural e, por isso, são mais frequentemente aplicados em estudos ambientais. Em geral, os isótopos de $\mathrm{C}, \mathrm{N}$ e $\mathrm{S}$ são utilizados para elucidar vias fotossintéticas, processos fisiológicos e determinar os recursos alimentares assimilados, enquanto os isótopos de $\mathrm{H}$ e $\mathrm{O}$ são utilizados para determinar potenciais fontes de água (FRY, 2008; MARTINELLI et al., 2009).

A razão (R) entre dois isótopos estáveis (raro vs. abundante, como ${ }^{13} \mathrm{C} /{ }^{12} \mathrm{C}$ e ${ }^{15} \mathrm{~N} /{ }^{14} \mathrm{~N}$ ) é quantificada nas amostras analisadas e expressa em relação ao padrão internacional, com a notação $\left.\delta: \delta X \% o=\left[\left(\mathrm{R}_{\text {amostra }} / \mathrm{R}_{\text {padrão }}\right)-1\right)\right] * 1000$, onde $\mathrm{X}$ é o isótopo raro (e.g. $\delta^{13} \mathrm{C}$ ou $\delta^{15} \mathrm{~N}$ ). Quando uma amostra possui maior quantidade do isótopo raro em relação ao padrão internacional ela é denominada enriquecida (valores mais positivos de $\delta$ ). Por outro lado, amostras que possuem menor quantidade do isótopo raro em relação 
ao padrão internacional são denominadas empobrecidas (valores mais negativos de $\delta$ ) (FRY, 2008; MARTINELLI et al., 2009). Os estudos que tratam da composição isotópica de qualquer amostra são baseados nessas razões, determinadas analiticamente em um espectrômetro de massa de razão isotópica, ou "Isotope Ratio Mass Spectrometer" (IRMS). O processo no qual ocorre a discriminação de um isótopo em relação ao outro é denominado fracionamento isotópico, que resulta no enriquecimento ou empobrecimento do isótopo raro da amostra em relação a sua fonte.

Estudos pioneiros com isótopos estáveis em animais fizeram uso de dieta controlada e observaram que os recursos alimentares consumidos e assimilados pelos organismos se refletiram claramente em sua assinatura isotópica (DENIRO e EPSTEIN, 1978; 1981). Desde então, esses traçadores químicos são utilizados para investigar cadeias e redes alimentares, padrões de movimentação dos organismos, assimilação alimentar, posição trófica e fluxo de energia nos ecossistemas (e.g., FRY et al., 1999; DEEHR et al., 2014; DI BENEDITTO et al., 2017; WANG et al., 2018; 2020; FERREIRA et al., 2020; 2021).

No caso dos crustáceos decápodes, a aplicação da ferramenta isotópica para estudos sobre biologia, ecologia, manejo pesqueiro e conservação tem se expandido na última década. No entanto, para que novos estudos sejam direcionados com vistas ao preenchimento das lacunas do conhecimento sobre o tema, é necessário analisar o estado da arte das publicações disponíveis. Diante disso, este trabalho tem como objetivo apresentar análise bibliométrica sobre a utilização de isótopos estáveis em estudos com decápodes na última década (2010-2020).

\section{METODOLOGIA}

Este trabalho avaliou artigos científicos publicados em periódicos indexados e revisados por pares entre 2010 e 2020, que incluíram isótopos estáveis como ferramenta de estudo para crustáceos decápodes. As bases de busca bibliográfica utilizadas foram Google Scholar, Science Direct e Web of Science, e as palavras-chave (em inglês) foram: Crustacea, Decapoda, stable isotopes e isotopic ecology. Monografias, dissertações, teses, resumos apresentados em eventos científicos e relatórios técnicos não foram considerados no levantamento das referências devido à incerteza quanto à revisão por pares. As publicações foram classificadas como 'diretas', quando o objetivo principal do estudo 
estava relacionado aos decápodes, e como 'indiretas' quando os decápodes faziam parte da amostragem, mas não eram o alvo principal do estudo.

\section{RESULTADOS E DISCUSSÃO}

No total, foram levantados 440 artigos subdivididos em 208 publicações 'diretas' e 232 publicações 'indiretas' (Figura 1). Entre 2010 e 2020, houve aumento exponencial na aplicação de isótopos estáveis em estudos sobre decápodes $\left(\mathrm{R}^{2}=0,801\right)$. Nota-se que a partir de 2015 a quantidade de publicações que trataram direta e indiretamente do tema foram equiparáveis, o que reflete o incremento de estudos com foco direcionado aos decápodes (Figura 1).

Figura 1 - Número de artigos relacionados a aplicação de isótopos estáveis como ferramenta de estudo para crustáceos decápodes (2010-2020).

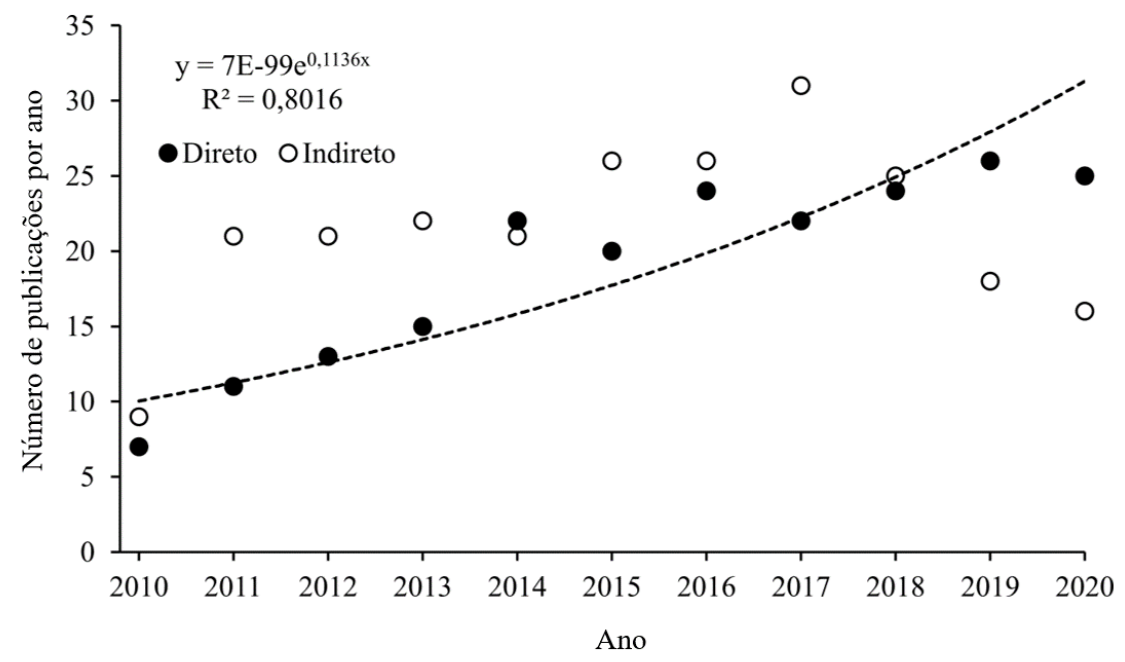

Fonte: Ferreira, K.A.

O cenário global relacionado a publicação de artigos que utilizaram isótopos estáveis como ferramenta de estudo para decápodes (apenas publicações 'diretas') indicou diferença entre os continentes. Os esforços foram concentrados, principalmente, na América do Norte seguida da Ásia, e o menor número de publicações esteve relacionado a América Latina e África (Figura 2). 
Figura 2 - Distribuição global da publicação de artigos relacionados a aplicação de isótopos estáveis como ferramenta de estudo para crustáceos decápodes (2010-2020).

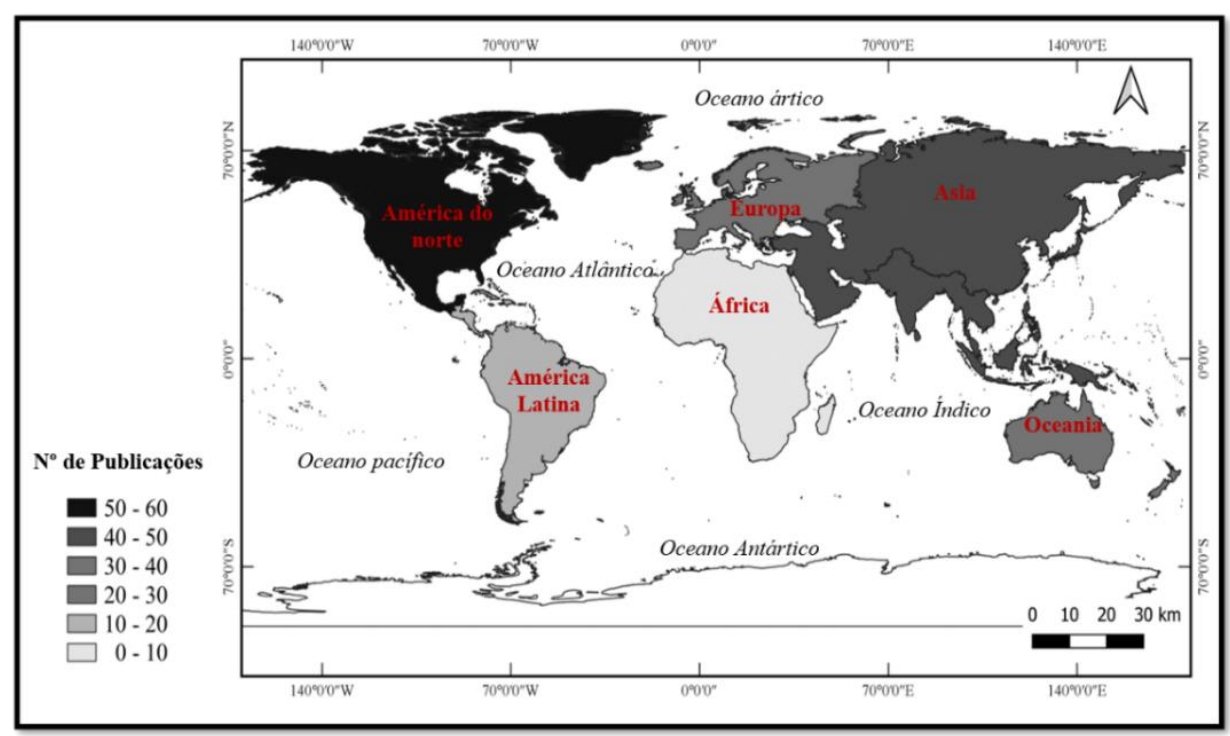

Fonte: Ferreira, K.A.

O exame dos mesmos dados por país mostra que a quantidade de publicações nos continentes foi impulsionada por esforços pontuais. Assim, entre 2010 e 2020, os Estados Unidos (EUA) lideraram as pesquisas na América do Norte, representando 49\% do total de artigos no continente. Em relação à Ásia, a China obteve $36 \%$ dos estudos, enquanto na Europa e na Oceania foram Espanha (20\%) e Austrália (97\%) que estiveram à frente, respectivamente. Na América Latina, o Brasil liderou com 33\% dos artigos publicados. $\mathrm{Na}$ África, a maior contribuição foi proveniente da África do Sul (37\%).

A liderança dos EUA nas publicações sobre isótopos estáveis em crustáceos se justifica não somente por questões relacionadas a economia forte e investimentos em ciência e tecnologia, mas também pelo seu pioneirismo na ciência isotópica, cujos primeiros estudos datam de meados do século XX. Por exemplo, Craig (1953) utilizou isótopos estáveis na compreensão de processos geoquímicos, Abelson e Hoering (1961) utilizaram essa ferramenta para compreender a formação de aminoácidos em organismos fotossintéticos. A relação entre a composição isotópica e a dieta dos animais foi investigada inicialmente pelos estudos de DeNiro e Epstein (1978) e Fry e Parker (1979).

Em geral, a distribuição dos artigos publicados sobre o tema segue o padrão econômico global, no qual os países mais ricos e com maiores investimentos em ciência e tecnologia lideram com maior número de publicações. Em 2018, por exemplo, os EUA e a China aplicaram US\$ 476,5 bilhões e US\$ 370,6 bilhões em pesquisa e 
desenvolvimento científico, respectivamente. Os dois países juntos somam $62 \%$ do investimento global e mais da metade do patenteamento mundial de tecnologias avançadas (disponível em: http://www.portal.sbpcnet.org.br/noticias/as-grandes-nacoesinvestem-em-ciencia-e-tecnologia-nao-por-serem-ricas-mas-sao-ricas-porque-investemem-ciencia-e-tecnologia-afirma-roberto-amaral/). Considerando que a ciência isotópica possui elevados custos operacionais, desde a montagem e manutenção da logística laboratorial até a realização das análises propriamente ditas, é natural que em países onde há maior investimento em ciência e tecnologia haja maior desenvolvimento neste aspecto e, consequentemente, maior número de artigos publicados.

Os artigos científicos indicaram 278 espécies de decápodes estudadas (menos de $2 \%$ do total), distribuídas nas subordens Dendrobranchiata e Pleocyemata. A subordem Dendrobranchiata possui duas superfamílias, Penaeoidea e Sergestoidea, e a primeira representou em torno de $20 \%$ do total de espécies de decápodes que foram alvo de estudos que utilizaram isótopos estáveis. A subordem Pleocyemata se divide em oito infraordens (Achelata, Anomura, Astacidea, Axiidea, Brachyura, Caridea, Gebiidea e Polychelida), e a maior parte dos estudos com isótopos estáveis envolveram Brachyura (38\%), Caridea $(21 \%)$ e Anomura (7\%).

A maior concentração dos estudos em Penaeoidea, Brachyura, Caridea e Anomura se justifica pela importância comercial dos crustáceos que representam esses táxons, conforme dados de estatísticas pesqueiras (FAO, 2016). Espécies de Penaeoidea e Caridea, por exemplo, são importantes alvos de pescarias de camarões em águas tropicais e temperadas, respectivamente. No caso dos caranguejos, as espécies com maior relevância comercial pertencem a Brachyura (caranguejos verdadeiros) e Anomura (caranguejos eremitas e caranguejos porcelanídeos). Dessa forma, há grande interesse em conhecer a ecologia desses organismos para sua conservação e manutenção das populações em níveis viáveis de exploração comercial.

Nos artigos levantados, a ferramenta isotópica foi aplicada principalmente no entendimento da ecologia trófica de decápodes. Nesse contexto, os artigos estavam relacionados a dieta e preferências alimentares, uso do habitat, metabolismo alimentar e interações tróficas (Tabela 1, Figura 3). Embora algumas temáticas em ecologia trófica estejam relacionadas entre si, tais como dieta e interações tróficas, optou-se por quantifica-las separadamente devido às particularidades que os autores propuseram nos estudos. Além dos artigos sobre ecologia trófica, verificou-se outras abordagens que 
fizeram uso de isótopos estáveis como ferramenta de estudo para decápodes, tais como conservação e gestão/manejo pesqueiro, e artigos relacionados a ajustes metodológicos para aprimorar a interpretação dos resultados isotópicos (Tabela 1; Figura 3).

Tabela 1 - Temas abordados nos artigos que utilizaram isótopos estáveis como ferramenta de estudo para crustáceos decápodes (2010-2020).

\begin{tabular}{|c|c|c|}
\hline Abordagem & Temática & Descrição \\
\hline \multicolumn{3}{|l|}{$\begin{array}{l}\text { Ecologia } \\
\text { trófica }\end{array}$} \\
\hline & Dieta & $\begin{array}{l}\text { Assimilação dos recursos alimentares no habitat; } \\
\text { Dieta experimental }\end{array}$ \\
\hline & Habitat & $\begin{array}{l}\text { Movimentação dos organismos e das populações; } \\
\text { Utilização dos recursos (costeiro, oceânico, pelágico, } \\
\text { bentônico) } \\
\text { Sítios alimentares preferenciais }\end{array}$ \\
\hline & Metabolismo & Mudanças na dieta e alterações metabólicas \\
\hline & $\begin{array}{l}\text { Interações tróficas, } \\
\text { incluindo cadeias e } \\
\text { redes alimentares }\end{array}$ & $\begin{array}{l}\text { Interações intra- e interespecíficas; } \\
\text { Nicho trófico; } \\
\text { Papel trófico em cadeias e teias alimentares }\end{array}$ \\
\hline \multicolumn{3}{|l|}{ Outras } \\
\hline & Conservação & $\begin{array}{l}\text { Trajetória trófica de compostos e poluentes } \\
\text { antropogênicos; } \\
\text { Influência de espécies invasoras/introduzidas nas } \\
\text { cadeias/redes alimentares nativas }\end{array}$ \\
\hline & Recurso pesqueiro & $\begin{array}{l}\text { Determinação de origem geográfica e autenticação; } \\
\text { Gestão/manejo pesqueiro }\end{array}$ \\
\hline & Ajustes metodológicos & $\begin{array}{l}\text { Determinação experimental de fracionamento } \\
\text { isotópico; } \\
\text { Comparação de fatores de discriminação isotópica }\end{array}$ \\
\hline
\end{tabular}

Fonte: Ferreira, K.A.

Os isótopos $\delta^{13} \mathrm{C}$ e $\delta^{15} \mathrm{~N}$ foram os mais utilizados nos artigos levantados, e alguns estudos utilizaram marcadores químicos complementares, tais como: ácidos graxos, mercúrio e/ou combinação com outros isótopos estáveis. Na última década, os isótopos estáveis foram aplicados principalmente em estudos de dieta e interações tróficas dos crustáceos decápodes, e as duas temáticas representaram juntas cerca de $47 \%$ das publicações levantadas neste trabalho (Figura 3). Os organismos consumidores adquirem suas composições isotópicas a partir da dieta (assimilação de alimento), refletindo seu habitat trófico. Assim, quaisquer alterações no habitat são refletidas nas assinaturas isotópicas dos consumidores (FRY et al., 2003). O fracionamento do isótopo de $\delta^{13} \mathrm{C}$ é pequeno, $0 \%$ a $1 \%$, e é utilizado para definir as fontes de energia (POST, 2007). Por 
outro lado, a razão isotópica de $\delta^{15} \mathrm{~N}$ dos consumidores são tipicamente mais enriquecidas, de 2\% a 4\% por nível trófico (PETERSON e FRY, 1985; FRY, 2003).

Figura 3 - Número de artigos que utilizaram isótopos estáveis como ferramenta de estudo para crustáceos decápodes. Os dados estão organizados em ordem decrescente da quantidade de publicações, e foram considerados apenas as publicações 'diretas' entre 2010 e 2020.

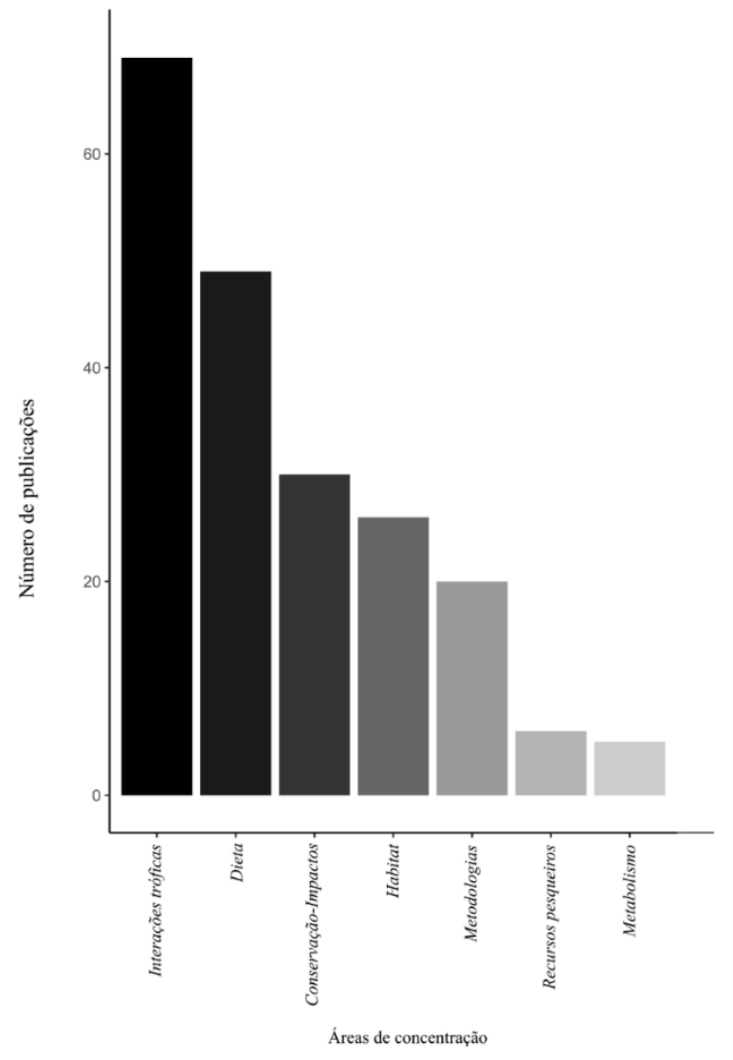

Fonte: Ferreira, K.A.

O conhecimento sobre os recursos alimentares consumidos e assimilados pelos decápodes permite compreender suas necessidades nutricionais e interações com outras espécies, o que é importante para avaliar sua história natural, comportamento, uso do habitat e processos ecológicos nos quais as espécies estão envolvidas (ABED-NAVANDI e DWORSCHAK, 2005; GUTIÉRREZ et al., 2016). Esse tipo de conhecimento permite avaliar como os decápodes influenciam o ambiente (e outras espécies) e como são afetados por ele.

Um aspecto importante no entendimento das relações alimentares a partir da ferramenta isotópica é a quantificação do fracionamento isotópico entre fontes alimentares e consumidores, e entre os tecidos corporais. No entanto, ainda há poucas pesquisas experimentais que visam compreender esse processo de fracionamento 
isotópico em tecidos de crustáceos decápodes (MAZUMDER et al., 2018). Os fatores de discriminação isotópica são fundamentais em estudos de conectividade em redes alimentares, pois permitem a correção dos valores isotópicos dos consumidores para elaboração de modelos de mistura preditivos da assimilação alimentar (PHILLIPS e GREGG, 2003). Quando os fatores de discriminação não são compreendidos ou são malempregados, há imprecisão nas predições e aumento da incerteza, o que fragiliza a aplicabilidade dos modelos de mistura (PHILLIPS e KOCH, 2002; MARTÍNEZ DEL RIO et al., 2009; BOND e DIAMOND, 2011). Dessa forma, considerando que os decápodes desempenham papel ecológico importante no ambiente aquático, viabilizando parte da energia do ambiente bentônico aos níveis tróficos superiores das cadeias e redes alimentares, é preciso compreender os processos de discriminação isotópica nos diferentes tecidos corporais para que os modelos representem melhor as interações ecológicas no ambiente.

Os decápodes que se distribuem em águas continentais e costeiras são muito afetados pelos impactos antrópicos nessas regiões, que têm aumentado a cada ano devido aos crescentes processos de ocupação e industrialização (FRY et al., 2016). Dentre os ambientes costeiros mais afetados destacam-se os estuários, que são áreas de ocorrência e desenvolvimento de muitas espécies de decápodes. O carreamento de nitrogênio de origem antrópica para os estuários aumentou globalmente (LOTZE et al., 2006; MAZUMDER et al., 2015). A elevação dos níveis de nitrogênio em águas estuarinas estimula o crescimento de algas, alterando a produção primária das cadeias e redes alimentares locais (COLE et al., 2004; ELLIOT e QUINTINO, 2007), o que afeta não somente os decápodes, mas os demais organismos que ocorrem nesse ambiente.

Os crustáceos decápodes que se distribuem em regiões afetadas pela poluição podem ser utilizados como biomonitores a partir da determinação e do acompanhamento de variações na sua composição isotópica. Neste sentido, os isótopos estáveis são traçadores eficientes na identificação de fontes antropogênicas enriquecidas em nitrogênio, assim como na detecção de fontes primárias de $\delta^{13} \mathrm{C}$ decorrentes de alterações ambientais (EYRE et al., 2002; MUNROE et al., 2018). O isótopo $\delta^{18} \mathrm{O}$ em combinação com elementos traço e $\delta^{15} \mathrm{~N}$ se mostrou eficaz em avaliações da poluição em ecossistemas costeiros e seus reflexos nos decápodes (FRY et al., 2016). Assim, a utilização de espécies de crustáceos é reconhecida como eficaz para monitorar a qualidade dos ambientes 
aquáticos devido à sua capacidade em acumular poluentes provenientes da água, sedimento e/ou fontes alimentares (COLIN et al., 2016).

A técnica de isótopos estáveis é eficaz em fornecer informações sobre a rastreabilidade e autenticidade de produtos alimentares, permitindo sua aplicação na gestão pesqueira. A aquicultura é considerada a nova fronteira para o aumento mundial da produção de proteína animal e da segurança alimentar, pois mantém a perspectiva de reduzir a pressão sobre os estoques sobrexplotados pelas pescarias extrativas (FAO, 2016). Organismos cultivados são influenciados por diferentes regimes alimentares e ambientes em comparação com organismos pescados no habitat natural. Essas diferenças levam às composições isotópicas distintas e, consequentemente, a rastreabilidade dos produtos (ARECHAVALA-LOPEZ et al., 2013; VINCI et al., 2013; GAMBOADELGADO et al., 2014; LI et al., 2018; FERREIRA et al., 2021). Os isótopos estáveis comumente utilizados na autenticação e rastreabilidade são ${ }^{13} \mathrm{C} /{ }^{12} \mathrm{C},{ }^{18} \mathrm{O} /{ }^{16} \mathrm{O},{ }^{2} \mathrm{H} /{ }^{1} \mathrm{H}$, ${ }^{15} \mathrm{~N} /{ }^{14} \mathrm{~N} \mathrm{e}{ }^{34} \mathrm{~S} /{ }^{32} \mathrm{~S},{ }^{11} \mathrm{~B} /{ }^{10} \mathrm{~B}$ e ${ }^{87} \mathrm{Sr} /{ }^{86} \mathrm{Sr}$ (LI et al., 2016).

A análise das razões ${ }^{13} \mathrm{C} /{ }^{12} \mathrm{C}$ e ${ }^{15} \mathrm{~N} /{ }^{14} \mathrm{~N}$ demonstrou a viabilidade de avaliações isotópicas para distinguir camarões capturados na natureza daqueles provenientes de fazendas de aquicultura. Essas informações são fundamentais para autenticar o método de produção de diversos produtos da pesca (GAMBOA-DELGADO et al., 2014). No entanto, a produção de organismos cultivados pode representar impacto antrópico sobre as populações nativas, pois amplia a entrada de recursos alóctones nos ecossistemas aquáticos. A introdução de insumos ricos em nutrientes (rações e afins) e de espécies invasoras pode alterar a estrutura e o funcionamento das cadeias e redes alimentares locais (SATO e WATANABE, 2013; BAŠIĆ et al., 2015). Essa alteração se reflete na composição isotópica das espécies nativas, conforme demonstrado em Izquierdo-Gomez et al. (2015) para decápodes.

A pesca exerce papel fundamental na sociedade como fonte de emprego, renda e nutrição, e é responsável por cerca de $25 \%$ da proteína animal consumida no mundo (GUTIÉRREZ et al., 2011). No entanto, os impactos provocados pela pesca, tais como sobrepesca, captura incidental de espécies não-alvo, e alterações do habitat podem ter graves consequências ao ecossistema aquático (DAYTON et al., 1995; ROBERTS et al., 2005). A técnica de isótopos estáveis tem potencial no fornecimento de informações da exploração pesqueira de decápodes, e pode servir como ferramenta para avaliar os impactos nas espécies exploradas (e.g. JACKSON et al., 2012; GAMBOA-DELGADO 
et al., 2014; MAZUMDER et al., 2015; ORTEA e GALHARDO et al., 2015; FRY et al., 2016; GORMAN et al., 2017; HINZ et al., 2017; LI et al., 2018; GOPI et al., 2019; MARTÍNEZ-DURAZO et al., 2019; GÜL \& GRIFTEN et al., 2020; TAO et al., 2020; YORIO et al., 2020; FERREIRA et al., 2021). Entretanto, esses estudos ainda não representam percentual elevado das abordagens realizadas na última década (Figura 3).

\section{CONCLUSÃO}

O número de publicações que utilizou isótopos estáveis como ferramenta de estudo para crustáceos decápodes progrediu na última década (2010-2020), mas ainda há assimetria entre as pesquisas e muitas lacunas a serem preenchidas. A disparidade na quantidade de estudos desenvolvidos entre os continentes (e países) chama atenção, e isso se explica pelas diferenças econômicas e de investimento em ciência e tecnologia, que não são de fácil solução. Entretanto, essa desproporção poderia ser minimizada se houvesse mais colaborações científicas entre grupos de pesquisa interessados nos crustáceos decápodes.

Outro aspecto que chama atenção é o baixíssimo percentual $(<2 \%)$ de espécies estudadas em comparação ao total de espécies. O foco dos estudos em decápodes de interesse comercial merece considerações. A utilização desses organismos como fonte de proteína alimentar e geradores de emprego e renda justifica esse interesse, ao mesmo tempo que negligencia a importância ecológica dos demais crustáceos que não possuem interesse econômico imediato.

A análise bibliométrica demonstrou que os estudos se concentraram no tema ecologia trófica, com base nos isótopos estáveis de carbono e nitrogênio. Obviamente que o entendimento dos aspectos ecológicos de decápodes, incluindo dieta e relações tróficas, é importante, até porque a minoria das espécies foi estudada até agora. No entanto, é fundamental que os estudos futuros contemplem outras áreas da aplicação dos isótopos estáveis, tais como conservação e gestão pesqueira. Esses temas são importantes para a manutenção das populações de decápodes no ambiente devido à degradação de suas áreas de uso, em especial águas continentais e costeiras, e a sobrexplotação decorrente da pesca extrativa. 


\section{AGRADECIMENTOS}

Os autores agradecem ao fomento recebido pelo Conselho Nacional de Desenvolvimento Científico e Tecnológico - CNPq (processo 301.259/2017-8), Fundação Carlos Chagas Filho de Amparo à Pesquisa do Estado do Rio de Janeiro - FAPERJ (processo E26/200.797/2021), e em parte pela Coordenação de Aperfeiçoamento de Pessoal de Nível Superior - Brasil (CAPES) - Código de Financiamento 001.

\section{REFERÊNCIAS}

ABED-NAVANDI, D.; DWORSCHAK, P.C. Food sources of tropical thalassinidean shrimps: a stable-isotope study. Marine Ecology Progress Series, v. 291, p. 159-168, 2005. https://doi.org/10.3354/meps291159

ABELSON, P.H.; HOERING, T.C. Carbon isotope fractionation in formation of amino acids by photosynthetic organisms. Proceedings of the National Academy of Sciences of the United States of America, v. 47, p. 623-632, 1961.

https://doi.org/10.1073/pnas.47.5.623

ABRANTES, K.G.; BARNETT, A.; BOUILLON, S. Stable isotope-based community metrics as a tool to identify patterns in food web structure in east African estuaries. Functional Ecology, v. 28, p. 270-282, 2014. https://doi.org/10.1111/13652435.12155

ARECHAVALA-LOPEZ, P.; FERNANDEZ-JOVER, D.; BLACK, K.D.; LADOUKAKIS, E.; BAYLE-SEMPERE, J.T.; SANCHEZ-JEREZ, P.; DEMPSTER, T. Differentiating the wild or farmed origin of Mediterranean fish: a review of tools for sea bream and sea bass. Reviews Aquaculture, v. 5, p. 137-157, 2013. https://doi.org/10.1111/raq.12006.

BAŠIĆ, T.; BRITTON, J. R.; JACKSON, M.C.; READING, P.; GREY, J. Angling baits and invasive crayfish as important trophic subsidies for a large cyprinid fish. Aquatic Sciences, v. 77, p. 153-160, 2015. https://doi.org/10.1007/s00027-016-0483-2

BOND, A.L., DIAMOND, A.W. Recent Bayesian stable-isotope mixing models are highly sensitive to variation in discrimination factors. Ecological Applications, v. 21, p. 1017-1023, 2011. https://doi.org/10.1890/09-2409.1

BRANCO, J.O., VERANI, J.R. Dinâmica da alimentação natural de Callinectes danae Smith (Decapoda, Portunidae) na Lagoa da Conceição, Florianópolis, Santa Catarina, Brasil. Revista Brasileira de Zoologia, v. 14, p. 1003-1018, 1997.

BRUSCA, R.C.; BRUSCA, G.J. Invertebrados. Rio de Janeiro: Guanabara Koogan, 2007.

BUI, T.H.H.; LEE, S.Y. Does 'you are what you eat' apply to mangrove grapsid crabs? PLoS One, v. 9, e89074, 2014. https://doi.org/10.1371/journal.pone.0089074 
COLE, M.L.; VALIELA, I.; KROEGER, K.D.; TOMASKY, G.L.; CEBRIAN, J.; WIGAND, C.; McKinney, R.A.; Grady, S.P.; CARVALHO DA SILVA, M.H. Assessment of a $\delta^{15} \mathrm{~N}$ isotopic method to indicate anthropogenic eutrophication in aquatic ecosystems. Journal of Environmental Quality, v. 33, p. 124-132. 2004. https://doi.org/10.2134/jeq2004.1240

COLIN, N.; PORTE, C.; FERNANDES, D.; BARATA, C.; PADRÓS, F.;

CARRASSÓN, M.; MACEDA-VEIGA, A. Ecological relevance of biomarkers in monitoring studies of macro-invertebrates and fish in Mediterranean rivers. Science of the Total Environment, v. 540, p. 307-323, 2016.

https://doi.org/10.1016/j.scitotenv.2017.12.316

CRAIG, H. The geochemistry of the stable carbon isotopes. Geochimica et Cosmochimica Acta, v. 3(2-3), p. 53-92, 1953. https://doi.org/10.1016/00167037(53)90001-5

DALL, W.; HILL, B.J.; ROTHLISBERG, P.C.; SHARPLES, D.J. The biology of the Penaeidae. In: BLAXTER, J.H.S.; SOUTHWARD, A.J. (Ed.). Advances in Marine Biology. London: Academic Press, 1990, p. 489-500.

DAYTON, P.K.; THRUSH, S.F.; AGARDY, M.T.; HOFMAN, R.J. Environmental effects of marine fishing. Aquatic conservation: marine and freshwater ecosystems, v. 5, p. 205-232, 1995. https://doi.org/10.1002/aqc.3270050305

DEEHR, R.A.; LUCZKOVICH, J.J.; HART, K.J.; CLOUGH, L.M.; JOHNSON, B.J.; JOHNSON, J.C. Using stable isotope analysis to validate effective trophic levels from Ecopath models of areas closed and open to shrimp trawling in CoreSound, NC, USA. Ecological Modelling, v. 282, p. 1-17, 2014.

https://doi.org/10.1016/j.ecolmodel.2014.03.005

DENIRO, M.J.; EPSTEIN, S. Influence of diet on the distribution of carbon isotopes in animals. Geochimica et Cosmochimica Acta, v. 42, p. 495-506, 1978.

DENIRO, M.J.; EPSTEIN, S. Influence of diet on the distribution of nitrogen isotopes in animals. Geochimica et Cosmochimica Acta, v. 45, p. 341-351, 1981.

DI BENEDITTO, A.P.M.; SICILIANO, S.; MONTEIRO, L.R. Herbivory level and niche breadth of juvenile green turtles (Chelonia mydas) in a tropical coastal area: insights from stable isotopes. Marine Biology, v. 164, 2017.

https://doi.org/10.1007/s00227-016-3044-2

ELLIOTT, M.; QUINTINO, V. The estuarine quality paradox, environmental homeostasis and the difficulty of detecting anthropogenic stress in naturally stressed areas. Marine Pollution Bulletin, v. 54, p. 640-645, 2007.

https://doi.org/10.1016/j.marpolbul.2007.02.003

EYRE, B.D.; MCKEE, L. J. Carbon, nitrogen, and phosphorus budgets for a shallow subtropical coastal embayment (Moreton Bay, Australia). Limnology and

Oceanography, v. 47, p. 1043-1055, 2002. https://doi.org/10.4319/lo.2002.47.4.1043 
FAO - FOOD AND AGRICULTURE ORGANIZATION OF THE UNITED

NATIONS. Statistics of Fisheries and Aquaculture Department, 2016. Available at: http://www.fao.org/fishery/statistics/global-production/query/en.

FERREIRA, K.A; MONTEIRO, L.R.; DI BENEDITTO, A.P.M. The niche of shrimp stocks (Xiphopenaeus kroyeri Heller, 1862) from southeastern Brazil: a stable isotope approach. Journal of Threatened Taxa, v. 12, p. 16173-16176, 2020.

https://doi.org/10.11609/jott.5961.12.9.16173-16176

FERREIRA, K.A.; BRAGA, A.A.; DI BENEDITTO, A.P.M. Can stable isotopes be applied to determine shrimp stocks origin in SE Brazil? An approach for utilization in fishery management. Ocean and Coastal Management, v. 205, 105500, 2021. https://doi.org/10.1016/j.ocecoaman.2020.105500

FRY, B.; PARKER, P.L. Animal diet in Texas seagrass meadows: $\delta^{13} \mathrm{C}$ evidence for the importance of benthic plants. Estuarine and Coastal Marine Science, v. 8, p. 499-509, 1979.

FRY, B. Stable Isotope Ecology. New York: Springer-Verlag, 2008.

FRY, B.; BALTZ, D.M.; BENFIELD, M.C.; FLEEGER, J.W.; GACE, A.; HAAS, H.L.; QUIÑONES-RIVERA, Z.J. Stable isotope indicators of movement and residency for brown shrimp (Farfantepenaeus aztecus) in coastal Louisiana marshscapes. Estuaries, v. 26, p. 82-97, 2003.

FRY, B.; CARTER, J.F.; TINGGI, U.; ARMAN, A.; KAMAL, M.; METIAN, M.; YACCUP, R.B. Prawn biomonitors of nutrient and trace metal pollution along AsiaPacific coastlines. Isotopes in environmental and health studies, v. 52, p. 619-632, 2016. https://doi.org/10.1080/10256016.2016.1149481

FRY, B.; MUMFORD, P.L.; ROBBLEE, M.B. Stable isotope studies of pink shrimp (Farfantepenaeus duorarum Burkenroad) migrations on the Southwestern Florida shelf. Bulletin of Marine Science, v. 65, p. 419-430, 1999.

GAMBOA-DELGADO, J.; MOLINA-POVEDA, C.; GODÍNEZ-SIORDIA, D.E.; VILLARREAL-CAVAZOS, D.; RICQUE-MARIE, D.E.; CRUZ-SUÁREZ, L.E. Application of stable isotope analysis to differentiate shrimp extracted by industrial fishing or produced through aquaculture practices. Canadian Journal of Fisheries and Aquatic Sciences, v. 71, p. 1520-1528, 2014. https://doi.org/10.1139/cjfas-2014-0005

GUTIÉRREZ, N.L.; HILBORN, R.; DEFEO, O. Leadership, social capital and incentives promote successful fisheries. Nature, v. 470, p. 386- 389, 2011. https://doi.org/10.1038/nature09689

IZQUIERDO-GOMEZ, D.; SANCHEZ-JEREZ, P.; BAYLE-SEMPERE, J.T.; LOADER, N.J.; DE LEANIZ, C.G. Effects of coastal fish farms on body size and isotope composition of wild penaeid prawn. Fisheries Research, v. 172, p. 50-56, 2015. https://doi.org/10.1016/j.fishres.2015.06.017 
LI, L.; REN, W.; DONG, S.; FENG, J. Investigation of geographic origin, salinity and feed on stable isotope profile of Pacific white shrimp (Litopenaeus

vannamei). Aquaculture Research, v. 49, p. 1029-1036, 2018.

https://doi.org/10.1111/are.13551

LI, L.; BOYD, C.E.; SUN, Z. Authentication of fishery and aquaculture products by multi-element and stable isotope analysis. Food Chemistry, v. 194, p. 1238-1244, 2016. https://doi.org/10.1016/j.foodchem.

LOTZE, H.K., LENIHAN, H.S., BOURQUE, B.J., BRADBURY, R.H., COOKE, R.G., KAY, M.C.; Kidwell, S.M.; Kirby, M.X.; Peterson, C.H.; Jackson, J.B. Depletion, degradation, and recovery potential of estuaries and coastal seas. Science, v. 312, p. 1806-1809, 2006. https://doi.org/10.1126/science.1128035

MARAFON-ALMEIDA, A.; SOUZA-CONCEIÇÃO, J.M.; PANDOLFO, P.S.V. Distribuição e abundância de larvas de três espécies de penaeídeos (Decapoda) na plataforma continental interna adjacente à Baía da Babitonga, Sul do Brasil. PanAmerican Journal of Aquatic Sciences, v. 3, p. 340-350, 2008.

MARTÍNEZ DEL RIO, C.; WOLF, N.; CARLETON, S.A.; GANNES, L.Z. Isotopic ecology ten years after a call for more laboratory experiments. Biological Reviews of the Cambridge Philosophical Society, v. 84, p. 91-111, 2009.

https://doi.org/10.1111/J.1469-185X.2008.00064.X

MARTINELLI, L.A.; OMETTO, J.P.H.B.; FERRAZ, E.S.; VICTORIA, R.L.; CAMARGO, P.B.; MOREIRA, M.Z. Desvendando questões ambientais com isótopos estáveis. São Paulo: Oficina de Textos, 2009.

MAZUMDER, D.; JOHANSEN, M.P.; FRY, B.; DAVIS, E. Muscle and carapace tissue-diet isotope discrimination factors for the freshwater crayfish Cherax destructor. Marine and Freshwater Research, v. 69, p. 56-65, 2018. https://doi.org/10.1071/MF16360

MAZUMDER, D.; SAINTILAN, N.; ALDERSON, B.; HOLLINS, S. Inputs of anthropogenic nitrogen influence isotopic composition and trophic structure in SE Australian estuaries. Marine Pollution Bulletin, v. 100, p. 217-223, 2015. https://doi.org/10.1016/j.marpolbul.2015.08.047

MUNROE, S.E.; COATES-MARNANE, J.; BURFORD, M.A.; FRY, B.A. benthic bioindicator reveals distinct land and ocean-based influences in an urbanized coastal embayment. PloS one, v. 13, e0205408, 2018. https://doi.org/10.1371/journal.pone.0205408

OLIVIER, A.; PINTO, T.K.; SANTOS, D.P.; D'INCAO, F. Dieta natural do siri-azul Callinectes sapidus (Decapoda, Portunidae) na região estuarina da Lagoa dos Patos, Rio Grande, Rio Grande do Sul, Brasil. Iheringia Série Zoologia, v. 96, p. 305-313, 2006. https://doi.org/10.1590/S0073-47212006000300006

ORTEA, I.; GALLARDO, J.M. Investigation of production method, geographical origin and species authentication in commercially relevant shrimps using stable isotope ratio 
and/or multi-element analyses combined with chemometrics: An exploratory analysis.

Food chemistry, v. 170, 145-153, 2015.

https://doi.org/10.1016/j.foodchem.2014.08.049

PETERSON, B.J.; HOWARTH, R.W.; GARRITT, R.H. Multiple stable isotopes used to trace the flow of organic matter in estuarine food webs. Science, v. 227, p. 13611363, 1985. https://doi.org/10.1126/science.227.4692.1361

PHILLIPS, D.L.; KOCH, P.L. Incorporating concentration dependence in stable isotope mixing models. Oecologia, v. 130, p. 114-125, 2002.

https://doi.org/10.1007/S004420100786

PHILLIPS, D.L.; INGER, R., BEARHOP, S., JACKSON, A.L., MOORE, J.W., PARNELL, A.C.; Semmens, B.X.; Ward, E.J. Best practices for use of stable isotope mixing models in food-web studies. Canadian Journal of Zoology, v. 92, p. 823-835, 2014. https://doi.org/10.1139/cjz-2014-0127

PHILLIPS, D.; GREGG, J. Source partitioning using stable isotopes: coping with too many sources. Oecologia, v. 136, p. 261-269, 2003. https://doi.org/10.1007/ S00442003-1218-3

POST, D.M.; LAYMAN, C.A.; ARRINGTON, D.A.; TAKIMOTO, G.;

QUATTROCHI, J.; MONTANA, C.G. Getting to the fat of the matter: models, methods and assumptions for dealing with lipids in stable isotope analyses. Oecologia, v. 152, p. 179-189, 2007. https://doi.org/10.1007/s00442-006-0630-х

ROBERTS, C.M.; HAWKINS, J.P.; GELL, F.R. The role of marine reserves in achieving sustainable fisheries. Philosophical Transactions of the Royal Society B: Biological Sciences, v. 360, p. 123-132, 2005. https://doi.org/10.1098/rstb.2004.1578

RUPPERT, E.E.; FOX, R.S.; BARNES, R.D. Zoologia dos invertebrados: uma abordagem funcional-evolutiva. São Paulo: Roca, 2005.

SATO, T.; WATANABE, K. Do stage-specific functional responses of consumers dampen the effects of subsidies on trophic cascades in streams? Journal of Animal Ecology, v. 83, p. 907-915, 2014. https://doi.org/10.1111/1365-2656.12192

TOM, M.; GOREN, M.; OVADIA, M. The benthic phase of the life cycle of Parapenaeus longirostris (Crustacea, Decapoda, Penaeidae) along the Mediterranean coast of Israel. Hydrobiologia, v. 169, p. 339-352, 1988.

VINCI, G.; PRETI, R.; TIERI, A.; VIERI, S. Authenticity and quality of animal origin food investigated by stable-isotope ratio analysis. Journal of the Science of Food and Agriculture, v. 93, p. 439-448, 2013. https://doi.org/10.1002/jsfa.5970

WANG, S.; LUO, B.K.; QIN, Y.J.; SU, L.H.; STEWART, S.D.; WANG, T.T.; TANG, J.P.; HE, B.D.; ZHANG, J.H.; LIN, H.J.; YANG, Y. Consumer-diet discrimination of $\delta 13 \mathrm{C}$ and $\delta 15 \mathrm{~N}$ : Source-and feeding-oriented patterns based on gut content analysis in a large subtropical river of China. River Research and Applications, v. 36, p. 11241136, 2020. https://doi.org/10.1002/rra.3644 
WANG, S.; WANG, L.; CHANG, H.Y.; LI, F.; TANG, J.P.; ZHOU, X.A.; YANG, Y. Longitudinal variation in energy flow networks along a large subtropical river, China. Ecological Modelling, v. 387, p. 83-95, 2018.

https://doi.org/10.1016/j.ecolmodel.2018.08.019

Recebido em: 15/09/2021

Aprovado em: 15/10/2021

Publicado em: 20/10/2021 